\title{
OCULAR ALLERGY IN HANDLERS OF STREPTOMYCIN *
}

\author{
BY \\ J. Charamis \\ ATHENS
}

THE successful results obtained in the treatment of tuberculosis (particularly tuberculous meningitis) by streptomycin, and the low toxicity of this drug have encouraged its widespread use. Special streptomycin centres have been established, and we apply streptomycin treatment on a large scale. Toxic effects of streptomycin are very few nowadays. This must be attributed to the improved purity of the drug and to the wide experience which has been gained in its use. The toxic effects either involve the nervous system and the organs of the special senses or appear as allergic toxic disturbances.

Dermatitis from contact with streptomycin has been noted, chiefly in physicians and nurses who give many injections daily. Since 1947, first in the United States and later in England and France, studies have been published on pathological appearances of the skin and mucous membrane (conjunctiva) of hospital nurses. The study of such cases has proved that the condition was an allergic manifestation of sensitivity to streptomycin. Contact with the drug is either direct, during its preparation or injection, or indirect, from continuous contact with patients and their bedding.

There is no proof that sex plays any part in the appearance of allergy. Nurses seem to be more often affected because their work brings them into closer contact with the drug and with the patients during treatment. No definite allergic tendency is necessary before the appearance of dermatitis. The intra-dermal tests with weak solutions of streptomvcin, administered at frequent intervals to nurses, become strongly positive. This confirms the specific allergic sensitivity as soon as the first cutaneous or ophthalmic (conjunctival) signs appear. This specific drug-allergy appears after a period varying from one to six months of continuous. contact with streptomycin.

Sometimes general allergic symptoms such as dizziness, headache and fatigue may be noted. These are followed by the manifestation of dermatitis proper which appears mainly on the hands and face. The eyes and their adnexa are most frequently involved. Varying forms and intensities of blepharitis and conjunctivitis appear. First may come the familiar disturbances of blepharo-conjunctivitis,

\footnotetext{
* Received for publication, March 30, 1949.
} 
i.e., itching, epiphora and injection of the conjunctival vessels, and blepharitis itching provokes an eczematous dermatitis which may become-septic. The conjunctivitis sometimes becomes subacute or acute, and the conjunctiva and eyelids are very swollen. Sub-acute or chronic forms of this allergic or professional blepharoconjunctivitis may be found.

The local treatment should be mild and symptomatic. Chief attention should be given to the avoidance of any contact with streptomycin or with patients receiving streptomycin treatment. The period required for the cure of this benign form of allergic dermatitis is variable. Relapses of completely cured cases of allergic blepharitis and conjunctivitis mean that there has been a fresh contact with the drug.

Since the allergic sensitisation of the skin is not general, the selection of an ophthalmic location may be explained either by the immediate contact of the evelids (through contamination by the hands) with the solution of streptomycin during preparation or by the exposure of the worker's face to the products of evaporation of the drug. Allergic symptoms in persons working with streptomycin appear to be frequent, and according to present statistics involve from 5 to 57 per cent. of those handling the drug. Some protection may be obtained by the use of rubber gloves and protective goggles.

Many cases of this allergic blepharitis and conjunctivitis have already appeared in the special. streptomycin centres, e.g., of 110 nurses and two doctors who administered streptomycin intermittently or continuously thirty nurses (27 per cent.) showed allergic reactions. In 25 of them blepharitis and conjunctivitis appeared, and 22 had also dermatitis on the hands. The other 5 developed only dermatitis on the hands. Nurses who prepared solutions of streptomycin continuously over a long period and had allergic tendencies were more seriously affected.

The most severe of these cases was that of a nurse who had a general allergic tendency, and had prepared streptomycin continuously for two years. After she had been cured a severe dermatitis appeared on her hands and eyelids as soon as she began to work again with the drug: the skin reaction to streptomycin became strongly positive.

In another case of blepharitis and conjunctivitis an extensive dermatitis appeared on the exposed side of the neck.

The intra-dermal reactions to streptomycin in typical cases were generally positive, especially in the most severe and obstinate cases.

\section{REFERENCES}

Bernard, E.. Lotte, A. and Wolff, C. (1948).-Sem. hôp. Paris, 24, 2554.

Touraine, A. and Pichon, R. (1948). -Pr. méd., 56, 865.

Photinos, P. (1948).-Akademaiki Iatriki.

CHOREMIS, K. (1948). - Application of Streptomycin in the Treatment of Tuberculosis in Children. Athens.

Charocopos, S. et. al. (1948).-Arch. Greek Pathol. Soc., 229. 